\title{
Tolerancia a metales pesados a través del uso de micorrizas arbusculares en plantas cultivadas
}

\section{Heavy metal tolerance of crops by the use of arbuscular mycorrhiza fungi}
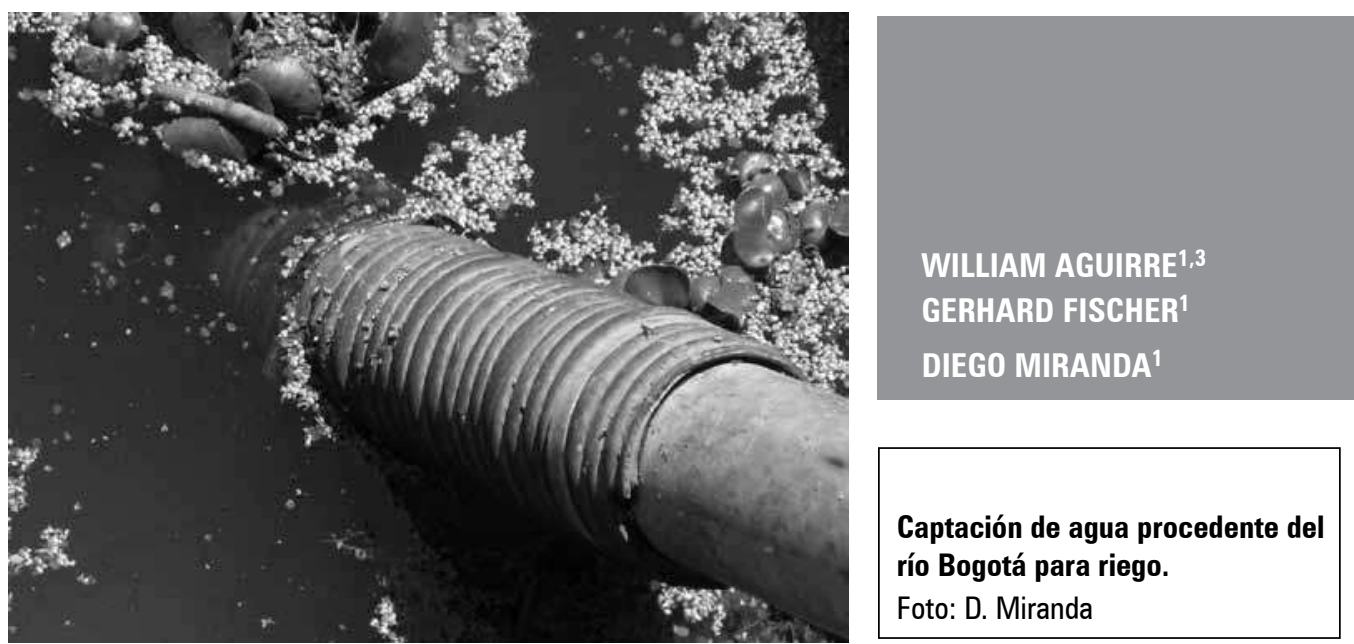

\section{RESUMEN}

La simbiosis establecida entre plantas y hongos formadores de micorrizas arbusculares (HFMA) ampliamente distribuida en ecosistemas y agroecosistemas, ha mostrado ser eficiente al disminuir la concentración de metales pesados (MP) en diferentes especies vegetales, constituyéndose en una herramienta potencial para el manejo de plantas cultivadas bajo estas condiciones. La tolerancia a esta toxicidad, puede variar según el metal pesado que se trabaja, la concentración del mismo en el suelo, las especies involucradas (hongo y vegetal) y las condiciones específicas de cada cultivo (bióticas y abióticas). Este artículo hace una revisión sobre los diferentes mecanismos fisiológicos de absorción, asimilación, translocación y acumulación de MP involucrados a nivel celular en la simbiosis, justificando el uso de micorrizas en la mitigación y tolerancia a esta contaminación.

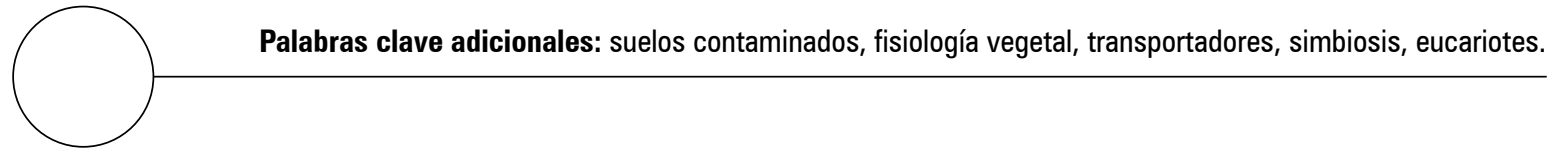

Facultad de Agronomía, Departamento de Agronomía, Universidad Nacional de Colombia, Bogotá (Colombia). Autor para correspondencia.wdaguirrer@unal.edu.co 


\section{ABSTRACT}

The symbiosis between plants and mycorrhiza fungi, established in ecosystems and agricultural environments throughout the planet, has been shown to efficiently reduce the concentration of heavy metals in plants, and has proved itself as a useful tool for the management of plants growing under these conditions. The conclusion is that tolerance to toxicity varies as a result of which heavy metal is present, it's concentration in the soil, the species involved (plant and fungi) and specific crop conditions (biotic and abiotic). The different physiological mechanisms of transport, absorption, assimilation and accumulation of heavy metals involved at the cellular level in this symbiosis are reviewed in this article, with a finding that the use of mycorrhiza in the mitigation of and tolerance to heavy metal contamination is justified.

Additional key words: polluted soils, plant physiology, transporters, symbiosis, eukaryotes.

Fecha de recepción: 05-01-2011

Aprobado para publicación: 27-05-2011
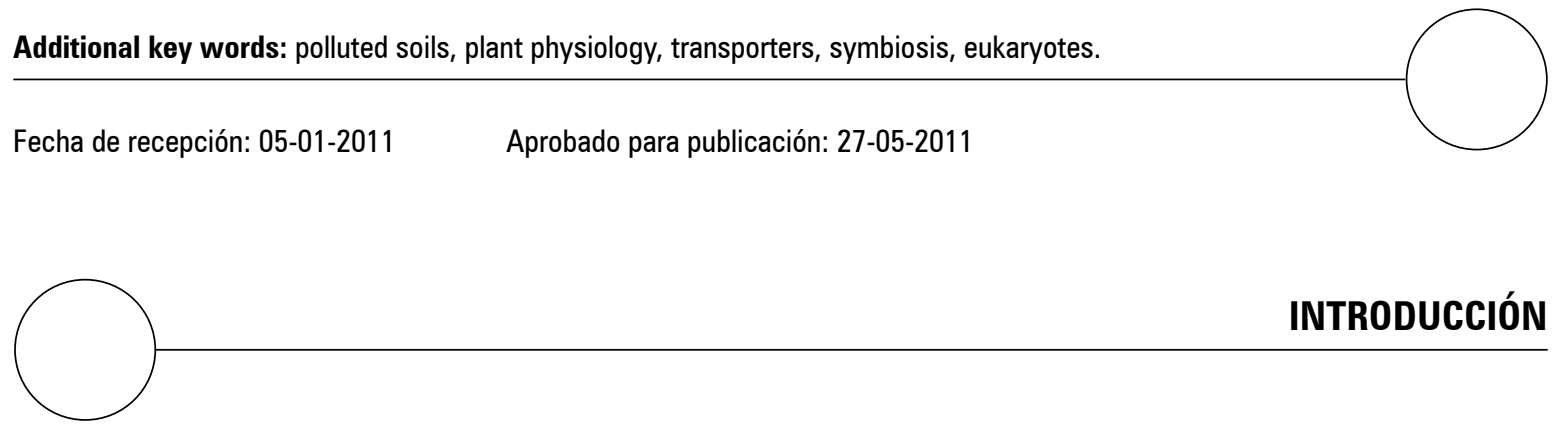

INTRODUCCIÓN

Uno de los problemas ambientales más importantes del planeta es la contaminación por metales pesados (MP), debido a la diversidad de fuentes de las que provienen (minería, industria de producción, aplicación de fertilizantes y agroquímicos, etc.), convirtiendo a los MP en amenaza también para la salud humana (Wang y Chen, 2009; Ortega-Larocea et al., 2007; Vega, 1988). Se habla de tres grupos de MP de importancia, el primer grupo incluye el $\mathrm{Hg}, \mathrm{Cr}, \mathrm{Pb}, \mathrm{Zn}, \mathrm{Cu}, \mathrm{Ni}$, $\mathrm{Cd}$, As, Co y Sn; el de metales preciosos (Pd, Pt, $\mathrm{Ag}, \mathrm{Au}, \mathrm{Ru}$, etc.) y los radionucleares (U, Th, Ra, Am, etc.). Sin embargo, aunque existen variadas clasificaciones que pueden o no compartir entre sí elementos considerados como MP, basadas en diferentes criterios específicos (Galán y Romero, 2008; Vega, 1988), suelen agruparse bajo este término todos los metales, excluyendo los alcalinos y alcalinotérreos, cuyo peso específico (densidad atómica) supera los 5 a $6 \mathrm{~g} \mathrm{~cm}^{-3}$ o que tienen un número atómico superior a 20 (Poschenrieder y Barceló, 2003).

La capacidad de algunos seres vivos (algas, bacterias, hongos, levaduras) para acumular elementos metálicos ha sido observada desde el punto de vista toxicológico (Khan, 2005); sin embargo, las últimas investigaciones indican que la biomasa inactiva o muerta de algunos microorganismos puede ligar iones metálicos a través de mecanismos fisicoquímicos (Wang y Chen, 2009). A pesar de que existe una gran variedad de materiales biológicos que pueden crear enlaces para retener iones metálicos, solamente aquellos cuya característica aparece en altas proporciones y mayor velocidad respecto al comportamiento y condiciones naturales, son seleccionados y considerado su uso, en diferentes ecosistemas (acuáticos y terrestres especialmente) (Wang y Chen, 2009).

En algunas especies de plantas cultivadas, la tolerancia a MP a través del uso de micorrizas arbusculares (MA), constituye un ejemplo de bioacumulación y biosorción, incluyendo mecanismos de intercambio, formación de complejos, coordinación, adsorción, interacción electrostática, quelación y precipitación, actuando individual o simultáneamente (Bainard et al., 2011; Wang y Chen, 2009; Sylvia et al., 2005; Unz y Shuttleworth, 1996). Variados estudios que reportan el uso de MA por sus beneficios a nivel nutricional y de activación de vías metabólicas de defensa en 
plantas, incluyen también, el efecto atenuante al estrés generado por contaminación con MP (Bainard et al., 2011), vinculando a nivel de géneroespecie, la alta capacidad que poseen para crear enlaces con $\mathrm{MP}$, que generalmente se presentan sin prioridad específica o como respuesta a algún grado de especificidad (Liao et al., 2003; Hildebrandt et al., 2007; Janoušková et al., 2006).

\section{LOS METALES PESADOS}

Los metales disponibles en el suelo son producto no solo de la naturaleza química del material parental sino del impacto antropogénico, componente singular en la biósfera (Wang y Chen, 2009; Galán y Romero, 2008; Entry et al., 2002). En el suelo, la movilidad de los MP en sus formas catiónicas, disminuye a medida que se incrementa el $\mathrm{pH}$, debido a que se precipitan formando hidróxidos, carbonatos o por conformar complejos orgánicos no asimilados por los organismos (Miranda et al., 2008a). Sin embargo, aquellos que pueden formar aniones como el uranio, estarán más disponibles para las plantas en suelos con $\mathrm{pH}$ alcalino, haciendo complejo su manejo. La fitotoxicidad producto de altos niveles de $\mathrm{MP}$, afecta el crecimiento y la formación de raíces secundarias, siendo crítica su acumulación e ingreso a la red trófica dadas las implicaciones que tiene sobre la salud humana (Poschenrieder y Barceló, 2003). El movimiento de los metales se da desde la solución del suelo a la raíz y posteriormente a través de las membranas de las células corticales. Luego al xilema y finalmente desde las hojas al floema (Acevedo et al., 2005), dependiendo del metal pesado en cuestión pues no todos siguen la misma ruta. Otro mecanismo de ingreso de sustancias potencialmente tóxicas para la planta, ocurre a nivel foliar, dependiendo de la morfología y tasa de respiración de la planta que permite su ingreso a partir de su acumulación y la variación en los gradientes de concentración. Allí, los metales provenientes de fuentes aéreas de contaminación, por ejemplo el $\mathrm{Pb}$, llegan a la planta producto de la deriva ocasionada por el viento. Igualmente, aplicaciones de fertilizantes de tipo foliar, posiblemente generan reacciones que facilitan la translocación de metales al interior del tejido. La capacidad de las plantas para acumular metales, varía según la especie vegetal y la naturaleza del contaminante (Janoušková et al., 2006; Entry et al., 2002). En el caso del cadmio (Cd), dada su alta solubilidad en suelo, se transloca a partir de las raíces a las partes aéreas de la planta, aumentando el riesgo de que sea incorporado a la cadena alimenticia debido a su similitud con el calcio $\left(\mathrm{Ca}^{2+}\right)$. Ambos iones poseen el mismo estado de oxidación y similares características iónicas, afectando diferentes procesos como la constitución de la pared celular y generando las consecuentes repercusiones de tener una pared celular debilitada en tejidos y órganos de la planta (Saldivar et al., 1997a). El Cd, inmovilizado en suelos por la materia orgánica y algunos microorganismos, se libera a través de ácidos orgánicos y otros exudados de la planta (Janoušková et al., 2006).

La contaminación por cromo (Cr), varía según su estado de oxidación. El $\mathrm{Cr}^{6+}$ es mucho más tóxico que el $\mathrm{Cr}^{3+}$ que se precipita en condiciones de $\mathrm{pH}$ alto, pero este último tiene gran capacidad de formar complejos con proteínas y aminoácidos (Khan, 2001; Albert, 1997). Asociado al metabolismo de la glucosa e influenciando su estado oxidativo por los cambios de $\mathrm{pH}$ y los exudados radicales (Khan, 2001), que aumentan o disminuyen su disponibilidad (caso maíz y tomate) (Acevedo et al., 2005) se acumula en las capas superficiales del suelo y se encuentra generalmente ligado con óxidos de hierro y materia orgánica, quedando solamente una pequeña porción en forma intercambiable. Sin embargo las formas hexavalentes se encuentran en forma de aniones lo que facilita su unión con materia orgánica y otros minerales tóxicos.

En plantas, la absorción de níquel (Ni) depende de las características físicoquímicas del suelo. Aquellos tratados con sedimentos y próximos a fuentes contaminantes, generan toxicidad en 
los ecosistemas (Miranda et al., 2008b). De alta movilidad, es considerado esencial para el metabolismo de las plantas y requerido en cantidades mínimas (0,001 $\left.\mathrm{mg} \mathrm{kg}^{-1}\right)$ (Ker y Charest, 2010). Se deposita en hojas y semillas, siendo común la clorosis como sintomatología asociada a altos niveles (Acevedo et al., 2005). En humanos, la dieta influye en la absorción del $\mathrm{Ni}$, ya que solo una porción se absorbe vía gastrointestinal, cuando se encuentra incorporado en alimentos. Sin embargo la absorción se eleva durante el ayuno, situación común en el sector rural (Rodríguez, 1991).

El mercurio $(\mathrm{Hg})$, principalmente en agua (inorgánico), pasa a compuestos orgánicos por acción de los microorganismos y de allí al suelo, plantas y animales, biomagnificándose en la cadena trófica (Gutiérrez et al., 1997). La probabilidad que el Hg depositado en suelo sea absorbido por las plantas, depende de las características del sistema edáfico ( $\mathrm{pH}$, material parental, oxido-reducción, drenaje, contenido materia orgánica, etc.) lo que determina su biodisponibilidad (Miranda et al., 2008a).

Para el caso del plomo $(\mathrm{Pb})$, el estrés generado incrementa y acelera la aparición de especies reactivas de oxígeno (ROS) que en altas cantidades afecta los mecanismos celulares, produciendo estrés oxidativo en plantas (Sharma, P. y R. Dubey, 2005). El suelo es su principal depósito, con altos niveles cerca a grandes ciudades y por deriva a causa del viento (hasta $2.400 \mu \mathrm{g} \mathrm{g}^{-1}$ ) y agua (Saldivar et al., 1997b; Badillo, 1987). La mayor parte es retenido por raíces con características de bioacumulación, específicamente cuando establecen relaciones simbióticas con MA (Vogél-Mikŭs et al., 2006), de especial importancia en cultivos irrigados con aguas provenientes de estas zonas.

\section{FISIOLOGÍA DE METALES EN EUCARIOTES}

Plantas y hongos, han desarrollado estrategias para la obtención de nutrientes de suelos con variada composición, usando diferentes mecanis- mos en la asimilación de metales, a la vez que previenen la toxicidad, coordinan transporte, quelación y secuestro de elementos metálicos a nivel celular, para mantener el equilibro iónico (Colangelo y Guerinot, 2006). Altas cantidades de metales pueden ser acumuladas en variedad de procesos independientemente de la ruta metabólica asociada, usando biomasa (viva-muerta), así como productos celulares (polisacáridos), en la remoción de MP (Andrade et al., 2010). Por esta razón, el genoma de eucariotes codifica varias familias de transportadores metálicos, que dirigen el proceso de translocación al interior de plantas y hongos, diferenciándose en ubicación a nivel celular, sustrato específico sobre el que actúan y patrones de expresión (Colangelo y Guerinot, 2006).

Las raíces de las plantas han desarrollado mecanismos que modifican su entorno mediante la excreción de compuestos orgánicos, como sustrato rico para la comunidad microbiana que las rodea (Sharma et al., 2007), facilitando la actividad del micro-ambiente y aumentando la disponibilidad de nutrientes presentes en el suelo (Silvia et al., 2005), involucrando dos aspectos básicos: quelación y acidificación de la rizósfera, lo que disminuye la movilidad de MP en exceso (González-Guerrero, 2005).

\section{La frontera de intercambio y los transportadores}

La pared celular constituye la primer barrera que debe atravesar cualquier compuesto que busque ingresar y su composición química varía de célula a célula (Wang y Chen, 2009), siendo característica su afinidad por cationes (alta densidad de cargas positivas), favoreciendo la retención de algunos MP en primera instancia.

Cuando se carece de pared celular, la membrana plasmática constituye la frontera para el intercambio, allí existen un gran número de transportadores encargados de controlar el ingreso y salida de diversas sustancias, con un alto grado de especificidad. a) Transportadores ZIP (zinc- 
iron regulated transported-like protein) introducen cationes divalentes en el citoplasma, siendo la naturaleza del ion transportado muy variada, pero incluyendo elementos como el Co, Mn, Zn, Fe y Cd. Su función orientada a la absorción de micronutrientes esenciales del medio exterior, moviliza al mismo tiempo las reservas presentes al interior de la célula. Son los responsables de absorber parte del $\mathrm{Zn}$ incorporado a la célula (Colangelo y Guerinot, 2006; González-Guerrero, 2005). b) Transportadores NRAMP (natural resistance associated macrophague protein), aumentan los cationes divalentes en el citoplasma, en especial Fe y $\mathrm{Mn}$, pero puede transportar también Zn, Co, Cu o Ni (González-Guerrero, 2005). c) Transportadores Ctr (Cu transporter), introducen $\mathrm{Cu}$ a la célula, formando una zona de baja densidad de electrones que actúa como poro específico para $\mathrm{Cu}$ y también influyen en su movilización desde la vacuola al citosol (Byung-Eun et al., 2008); sin embargo aún existen grandes vacios en el conocimiento de la mecánica de este trasnportador. Son regulados gracias a los niveles de $\mathrm{Cu}$ al interior de la célula (Colangelo y Guerinot, 2006; González-Guerrero, 2005). d) Transportadores CDF (cation difussion facilitator), reducen la concentración de cationes divalentes en el citoplasma, exportándolos al exterior de la célula o al interior de organelos, transportando también Zn, Co, Mn y Cd (Colangelo y Guerinot, 2006; González-Guerrero, 2005). e) Transportadores de tipo primario $A B C$ (ATP binding cassette), utilizan la hidrólisis de ATP para movilizar sustancias que incluyen lípidos, péptidos, azúcares y complejos de glutatión, siendo la sub-familia $M R P$, la encargada del transporte de MP a la vacuola o al exterior de la célula, a partir de los complejos que forman los MP con glutatión o los complejos formados con fitoquelatinas (Rodríquez-Serrano et al., 2008). f) ATPasas del tipo P, superfamilia de transportadores primarios de cationes, especialmente $\mathrm{H}, \mathrm{Ca}, \mathrm{Mg}, \mathrm{Na}, \mathrm{Zn}$, o Cu. En eucariotes, la sub-familia $C P x$ es la mejor estudiada en el transporte de metales por su asociación a bajos niveles de $\mathrm{Cu}$ en la sangre (transtornos de Melkes y Wilson). Estas ATPasas transportan activamente el $\mathrm{Cu}$, disminuyendo los niveles del metal en el citosol (Colangelo y Guerinot, 2006; González-Guerrero, 2005). g) Transportadores YSL (proteínas yellow stripe1-like), la más reciente identificada hasta el momento y cuyo estudio sigue en progreso, encargada del transporte de metales que han formado complejos a través de fitometalóforos y de la movilización de Fe, Zn, $\mathrm{Mn}$ y $\mathrm{Cu}$ especialmente (Colanguelo y Guerinot, 2006).

A nivel citoplasmático, la quelación prima en el papel de la retención de MP, ya sea para direccionamiento o reserva, mediante el uso de péptidos o proteínas que se encuentran al interior de la célula (González-Guerrero, 2005). El glutatión asociado al potencial redox del citoplasma, es fundamental en la eliminación de compuestos tóxicos mediante el proceso de conjugación. Posee la capacidad de unirse a cationes divalentes

Tabla 1. Transportadores involucrados en la homeostasis de MP (González-Guerrero, 2005).

\begin{tabular}{|c|l|l|}
\hline $\begin{array}{c}\text { Familia } \\
\text { transportadores metálicos }\end{array}$ & Elemento transportado & \multicolumn{1}{c|}{ Ubicación en célula } \\
\hline ATPasas & $\mathrm{Zn}, \mathrm{Cu}, \mathrm{Mg}$ & Membrana tilacoide y membrana citoplasmática \\
\hline ZIP & $\mathrm{Zn}, \mathrm{Fe}, \mathrm{Mn}, \mathrm{Co}, \mathrm{Cd}$ & Membrana citoplasmática \\
\hline CDF & $\mathrm{Zn}, \mathrm{Co}, \mathrm{Mn}, \mathrm{Cd}$ & Membrana vacuola y membrana citoplasmática \\
\hline $\mathrm{ABC}$ & $\mathrm{Cd}$ & Vacuola \\
\hline NRAMP & $\mathrm{Mn}, \mathrm{Fe}, \mathrm{Co}, \mathrm{Ni}, \mathrm{Zn}, \mathrm{Cu}$ & Membrana vacuola y membrana citoplasmática \\
\hline $\mathrm{Ctr}$ & $\mathrm{Cu}$ & Membrana citoplasmática \\
\hline YSL & $\mathrm{Fe}, \mathrm{Zn}, \mathrm{Mn}, \mathrm{Cu}$ & Membrana citoplasmática \\
\hline
\end{tabular}


como el $\mathrm{Cd}^{2+}$, para formar complejos que son retirados del citoplasma a través de transportadores específicos (Yadav, 2010). A partir del glutatión, son sintetizadas fitoquelatinas (plantas, hongos y animales) que reguladas por $\mathrm{MP}$, permiten que en el citoplasma se empleen transportadores específicos para retirar la fitoquelatina unida al metal (Yadav, 2010)

Las metalotioneinas son proteínas de menor tamaño (6.000-7.000 Da), que poseen un alto contenido de cisteínas, que posibilitan la quelación de iones metálicos y la protección contra agentes oxidantes principalmente. Al parecer su acción solo se regula transcripcionalmente en presencia de variados estímulos como la presencia de MP. A diferencia del glutatión y las fitoquelatinas no se le conoce un transportador asociado, deduciendo que son acumuladas en el citoplasma (González-Guerrero, 2005). Requieren de otras proteínas, las metalochaperonas que sirven como impulsora del metal para que se transfiera con rapidez. En el caso del $\mathrm{Cu}$, se han identificado tres tipos de metalochaperonas distintas. Las primeras, ligadas a la ruta de secreción microsomal; las segundas, intervienen en la síntesis de las superóxido dismutasa (SOD) de $\mathrm{Zn}$ y Cu; y por último las que actúan en la síntesis de la citocromo-oxidasa mitocondrial (GonzálezGuerrero, 2005).

\section{FISIOLOGÍA DE LA SIMBIOSIS Y TOLERANCIA A METALES PESADOS}

La colonización de ecosistemas terrestres por parte de las plantas, se debe en gran parte a la asociación de estas con MA, siendo la colonización espacial, el beneficio al hospedero y la tolerancia ambiental (Powell et al., 2009), la explicación de su alta importancia en ecosistemas naturales y su amplia distribución en todo el planeta (Bonfante y Genre, 2008; Redecker, 2008; Sylvia et al., 2005; Remy et al., 1994; Morton, 1988; Walker, 1983).
La dinámica presente en suelos (humedad, temperatura, disponibilidad de nutrientes y otros microorganismos) dadas las condiciones naturales, prácticas culturales, degradación y contaminación como el caso de los MP, pueden afectar la formación y función de la simbiosis, ya sea, por efecto sobre el hongo o la planta (Kernaghan, 2005 ; Sylvia et al., 2005; Entry et al., 2002; Gehring y Whitham, 2002; Miller, 2000; Miller y Sharitz, 2000; Johnson et al., 1992; Lodge, 1989). Sin embargo, el exceso de agua no ha sido tan ampliamente considerado, a pesar de que existe información de la asociación en plantas acuáticas (Dhillion y Ampornpan, 1992; Clayton and Bagyaraj, 1984; Read et al., 1976). La temperatura óptima del suelo para favorecer la simbiosis es de $30^{\circ} \mathrm{C}$, presentando variantes entre 18 y $40^{\circ} \mathrm{C}$ (Matsubara y Harada, 1996; Schenck et al., 1975).

Las estructuras más importantes de las MA, involucradas en la simbiosis y tolerancia MP, son los arbúsculos, las vesículas y las hifas (figura 1). Los primeros, se originan a partir de la ramificación dicotómica repetida de una hifa al interior de una célula vegetal y hacen efectivo el intercambio de nutrientes (Guerrero, 1996). El hongo inicialmente crece en medio de las células corticales, pero rápidamente penetra en la pared celular de la célula hospedera, creciendo con ella. A medida que se da este crecimiento, la membrana celular de la célula hospedera sufre una invaginación que envuelve al hongo, creando un compartimiento apoplástico, allí se acumula material de alta complejidad molecular. Esta invaginación impide el contacto directo del citoplasma de la célula del hongo con el de la célula de la planta y permite una zona de intercambio de nutrientes, muy eficiente entre los simbiontes (Hamel y Plenchette, 2007).

Las vesículas, ensanchamientos terminales de las hifas, con forma ovoide, de pared delgada o gruesa, dentro y fuera de la raíz, acumulan reservas de carbono en forma de lípidos, se encuentran 


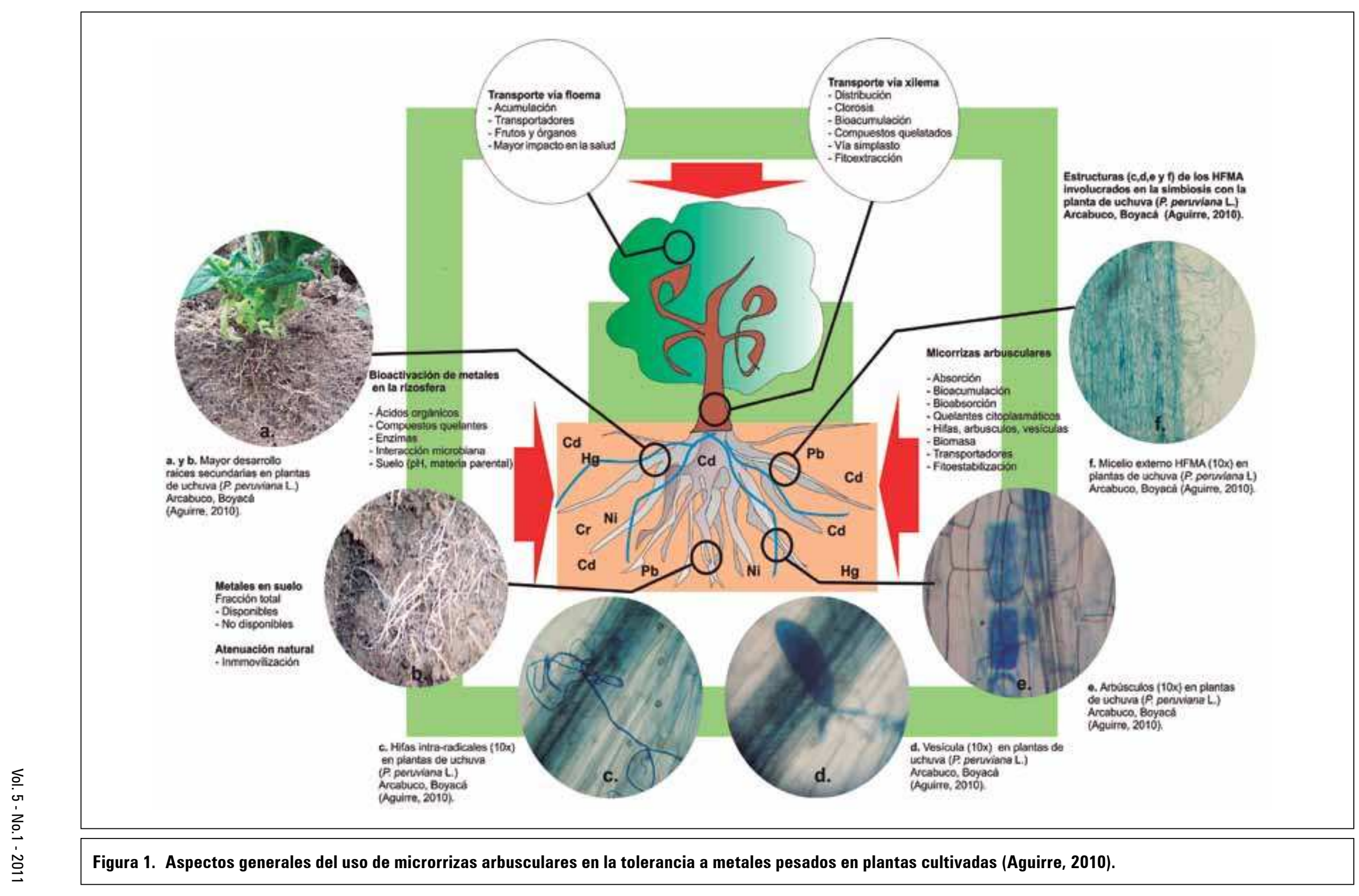


generalmente en espacios intercelulares pero también en espacios intracelulares. Su función principal es de almacenamiento, como compartimientos de MP (Sylvia et al., 2005). No son generadas por los géneros Gigaspora y Scutellospora (Guerrero, 1996).

Finalmente, las hifas, estructuras de colonización de las MA con características colonizadoras e infectivas (intraradicales) o exploradoras y de absorción de nutrientes (extraradicales) (Guerrero, 1996), emergen de la raíz y se extienden en el suelo varios centímetros, incluso metros, generando micelio externo como sistema de absorción de nutrientes y agua (Sylvia et al., 2005).

La glomalina, glicoproteína producida por las hifas de las micorrizas, ha mostrado su potencial al momento de establecer enlaces con moléculas de alta toxicidad como en el caso de los MP, lo que constituye una barrera eficaz que acumula metales en la masa micelial sin permitir que ingresen a las células vegetales (Hildebrandt et al., 2007; González-Guerrero, 2005).

Compuestos exudados por las raíces de las plantas (flavonoides, auxinas, strigolactona) permiten el reconocimiento de la micorriza, estimulando la germinación de esporas y el crecimiento y ramificación de las hifas (Akiyama et al., 2005; Vierheilig y Piché, 2002; Koske y Gemma, 1992; Gianinazzi-Pearson et al., 1989). Esto implica que la diversidad de las plantas y su edad (especialmente en cultivos perennes y semiperennes) desempeñan un papel importante en las comunidades de micorrizas en suelo, siendo remplazadas las especies de micorrizas según el estado fenológico del cultivo (Husband et al., 2002). En Colombia, existen reportes de altos niveles de dependencia entre el $\mathrm{pH}$ del suelo y la abundancia y diversidad de comunidades de MA dependiendo del género, indicando la no generalización de su aplicación a lo largo y ancho del país (Serralde y Ramírez, 2004).
La posibilidad de que las micorrizas sean heterocariotes, incluye que estos núcleos estén o no presentes en las nuevas generaciones de esporas, lo que implica que cada generación se constituye como un individuo genéticamente diferente a sus parentales, lo cual tiene gran influencia en la simbiosis y eficiencia de la asociación que se establece con las plantas (Hijri y Sanders, 2005; Pawlowska y Taylor, 2004; Larcher, 2003; Vandenkoornhuyse et al., 2002; Kuhn et al., 2001). Igualmente, al presentar hifas aseptadas hay alta probabilidad de que estos núcleos puedan moverse a través de ellas (Sanders y Croll, 2010).

Diferentes microorganismos son capaces de concentrar metales de manera activa y pasiva, en niveles que son substancialmente mayores a los del entorno que los rodea (Unz y Shuttleworth, 1996), razón por la cual, desde hace varias décadas existe un creciente interés por la interacción microorganismos-metales y sus posibles aplicaciones comerciales. La inmobilización de los MP mediante procesos activos (dependientes de energía) y pasivos (independientes de energía) se conocen con el nombre de bioacumulación y biosorción respectivamente, e incluyen los mecanismos mencionados anteriormente (quelación, intercambio iónico y encapsulamiento). Solo algunas plantas conocidas como metalófitas ( $M i$ nuarta veria, Armeria maritima spp., Thlaspi calaminare, Viola lutea spp., Alyssum wulfenianum, etc.) (Hildebrandt et al., 2007), poseen la capacidad de soportar condiciones adversas dadas por la contaminación causada por presencia de MP en el suelo en el que se desarrollan. Las metalófitas han desarrollado diversas adaptaciones fisiológicas entre las cuales se ha observado la asociación simbiótica con MA.

Las MA han demostrado de manera repetitiva su capacidad como agente reductor del estrés causado en plantas debido a la contaminación por MP en suelos (Hildebrandt et al., 2007). El mecanismo básico de funcionamiento de esta tolerancia es la expresión de diferentes genes que 
codifican para proteínas asociadas a variados mecanismos de transporte a nivel celular, que responden de manera diferente ante cada uno de los MP mencionados (Xin et al., 2010). Esta expresión génica es observada en los genes del micelio intra y extrarradical de modo diferenciado, como respuesta inicial al daño causado por las especies reactivas de oxígeno (ROS) siendo posiblemente el primer aporte de la simbiosis plantahongo (Hildebrandt et al., 2007). Se ha reportado que los MP que alcanzan el interior de las raíces son depositados en el tejido del parénquima, donde la mayoría de las estructuras del hongo (hifas intrarradicales, arbusculos y vesículas) se encuentran alojadas (Arriagada et al., 2010). Sin embargo la metodología asociada a los reportes consultados no permite la diferenciación del lugar de depósito de dichos metales (células vegetales o del hongo) (Hildebrandt et al., 2007).

Algunos organismos como las micorrizas ericoides cambian la composición estructural de su pared celular para responder a condiciones elevadas de $\mathrm{Zn}$ en su medio. Otros incorporan el MP a la estructura de hojas en las células epidérmicas, asociándolo a proteínas o silicatos (GonzálezGuerrero, 2005). A pesar de la información existente en este aspecto, el componente microbiológico, no ha sido lo suficientemente "integrado" al sistema de producción agrícola, contrario a las tendencias que en este aspecto que se llevan a nivel mundial (Ayres, 2008; Colemann, 2008; Barrios, 2007; Janviera et al., 2007; Schloter et al., 2003).

Es importante considerar en el uso de las MA el concepto de fitoestabilización, que se refiere a la inmobilización de MP o compuestos xenobióticos en suelo, aprovechando las características (fitoabsorción-fitoacumulación) que poseen estos microorganismos (Vörös y Takács, 2001; Takács et al., 2001). En las MA, la gran masa de micelio generada de manera natural gracias a las características de este simbionte, le confieren la posibilidad de acumular gran cantidad de compuestos xenobióticos y así limitar su disponibilidad para las plantas que se desarrollan en este sustrato. Al identificar los mecanismos de translocación de $\mathrm{MP}$ al interior de las células y su movimiento, es posible profundizar en las estrategias desarrolladas por las plantas que crecen bajo condiciones de extrema contaminación y así aplicarlas en otras de interés agrícola (Kochian, 2001; Yang et al., 2001; Verkleij et al., 2001; Hasegawa et al., 2001; Poschenrieder et al., 2001; Alcántara et al., 2001).

Otro efecto benéfico de la tolerancia a metales pesados a través del uso de MA, es el aumento y proliferación de raíces de tipo secundario (raicillas), con las cuales la planta simbionte, aumenta el área superficial de exploración lo que le permite mayor captación de elementos, incluyendo los MP (Smith et al., 2010).

\section{CONCLUSIONES}

El conocimiento de la fisiología y genética de cada integrante de esta simbiosis, es fundamental para el entendimiento del uso MA en la tolerancia a MP, en plantas cultivadas, siendo una opción viable dada las características de bioacumulación y biosorción presentes en eucariotes. Diversos estudios presentan la fitoestabilización, generada por el uso de las micorrizas como una buena solución de manejo a este problema de contaminación, pero se caracterizan por su gran distanciamiento en términos prácticos referidos a su uso en condiciones reales, siendo este un aspecto fundamental aún sin explorar. Sumado a lo anterior, para su aplicación, cada caso debe ser considerado como único e irrepetible, pues las características ambientales propias de cada cultivo expuesto a estas condiciones, así como los elementos involucrados (metal pesado, planta, ambiente, micorrizas) le infieren rasgos particulares y en constante dinámica, lo que incide en la efectividad de esta tolerancia. Es necesario ahondar en el conocimiento particular a nivel género y especie las MA en lo referente al metal que pueden bioacumular, siendo los estudios que aparecen en Colombia sobre 
especificidad y adaptación de estos organismos a diferentes condiciones de suelo, una primera aproximación para el mejor empleo de esta tecnología. Antes de que los beneficios del uso de MA en la recuperación de suelos con condiciones adversas tanto a nivel químico-físico sea generalizado, se hace necesario mejorar las características de desarrollo de las mismas bajo estas condiciones, aumentar el entendimiento de la diversidad genética de estos organismos y el rango en el que pueden actuar dada su capacidad fisiológica de adaptación.

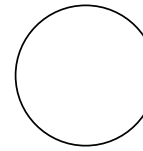

Acevedo, E., M. Carrasco, O. León, E. Martínez, P. Silva, G. Castillo, I. Ahumada, G. Borie y S. González. 2005. Criterios de calidad de suelo agrícola. Ministerio de Agricultura, Santiago.

Aguirre, W. 2010. Tolerancia a metales pesados a través del uso de micorrizas arbusculares en plantas cultivadas. Trabajo de grado. Facultad de Agronomía, Universidad Nacional de Colombia, Bogotá.

Akiyama, K., K. Matsuzaki y H. Hayashi. 2005. Plant sesquiterpenes induce hyphal branching in arbuscular mycorrhizal fungi. Nature 435, 824-827

Albert, L. 1997. Capítulo 14: El cromo. pp. 227-246. En: Palacios A. y L. América (eds.). Introducción a la toxicología ambiental. ECO, Metepec, México D.F.

Alcántara, E., A.M. Ginhas, M.A. Ojeda, M.J. Benítez y M. Benlloch. 2001. Metal accumulation by different plant species grown in contaminated media. pp. 460-461. En: Horst, W.J., M.K. Schenk, A. Bürkert, N. Claassen, H. Flessa, W.B. Frommer, H. Goldbach, H.W. Olfs, V. Römheld, B. Sattelmacher, U. Schmidhalter, S. Schubert, N. v. Wirén y L. Witenmayer (eds.). Plant nutrition-food security and sustainability of agro-ecosystems through basic and applied research. Kluwer, Dordrecht, The Netherlands.

Andrade, S., P. Gratão, R. Azevedo, A. Silveira, M. Schiavinato y P. Mazzafera. 2010.Biochemical and physiological changes in jack bean under mycorrhizal symbiosis growing in soil with increasing $\mathrm{Cu}$ concentrations. Environ. Exp. Bot. 68, 198-207.

Arriagada, C., G. Pereira, I. García-Romera y J.A. Ocampo. 2010. Improved zinc tolerance in Eucalyptus globulus inoculated with Glomus deserticola and Trametes versicolor or Coriolopsis rigida. Soil Biol.Biochem. 42, 118-124.

Ayres, R. 2008. Sustainability economics: Where do we stand? Ecol. Econ. 67, 281-310.

Badillo, J. 1987. Plomo. pp. 165-181. En: Palacios, A. y L. América. Curso básico de toxicología ambiental.

\section{REFERENCIAS BIBLIOGRÁFICAS}

Introducción a la toxicología ambiental. ECO, Metepec, México D.F.

Bainard, L., J. Klironomos y A. Gordon. 2011. The mycorrhizal status and colonization of 26 tree species growing in urban and rural environments. Mycorrhiza 21, 91-96.

Barrios, E. 2007. Soil biota, ecosystems services and land productivity. Ecol. Econ. 64, 269-285.

Bonfante, P. y A. Genre. 2008. Plants and arbuscular mycorrhizal fungi: an evolutionary develpmental perspective. Trends Plant Sci. 13 (9), 492-498.

Byung-Eun K., T. Nevitt y D. Thiele. 2008. Mechanisms for copper acquisition, distribution and regulation. Nat. Chem. Biol.4 (3), 176-185.

Clayton, J.S. y D.J. Bagyara. 1984. Vesicular-arbuscular mycorrhizas in submerged aquatic plants of New Zeal. Aquatic Bot. 19, 251-262.

Colanguelo, E. y M. Guerinot. 2006. Put the metal to the petal: metal uptake and transport throughout plants. Plant Biol. 9, 322-330.

Coleman, D. 2008. From peds to paradoxes: Linkages between soil biota and their influences on ecological process. Soil Biol. Biochem. 40, 271-289.

Dhillion, S.S. y L. Ampornpan. 1992. The influence of inorganic nutrient fertilization on the growth, nutrient composition and vesicular-arbuscular mycorrhizal colonization of pre-transplant rice (Oryza sativa L.) plants. Biol. Fertil. Soils 3, 85-91.

Entry, J.A., P.T. Rygiewicz, L.S. Watrud y P.K. Donnelly. 2002. Influence of adverse soil conditions on the formation and function of Arbuscular mycorrhizas. Adv. Environ. Res. 7, 123-138.

Galán, E. y A. Romero. 2008. Conferencia: Contaminación de suelos por metales pesados. En: http:// www.ehu.es/sem/macla_pdf/macla10/Macla10_48.pdf; consulta: septiembre de 2010. 
Gehring, C. y T. Whitham. 2002. Mycorrhizae-herbivore interactions: population and community consequences. En: van der Heijden, M. e I. Sanders (eds.). Mycorrhizal ecology. Ecological Studies 157, 295-316.

Gianinazzi-Pearson, V., B. Branzanti y S. Gianinazzi. 1989. In vitro enhancement of spore germination and early hyphal growth of a vesicular-arbuscular mycorrhizal fungus by host root exudates and plant flavonoids. Symbiosis 7, 243-255.

González-Guerrero, M. 2005. Estudio de los mecanismos implicados en la homeostasis de metales pesados en el hongo formador de micorrizas arbusculares Glomus intrarradices. Tesis de doctorado. Universidad de Granada, Granada, España.

Guerrero, E. 1996. Micorrizas: Recurso biológico del suelo. Fondo FEN Colombia, Bogotá.

Gutiérrez, M., L. Bucio y V. Souza. 1997. Capítulo 12: Mercurio. pp. 197-210. En: Palacios A., y L. América. 1997. Introducción a la toxicología ambiental. ECO, Metepec. México D.F.

Hamel, C. y C. Plenchette (eds.). 2007. Mycorrhizae in crop production. Haworth Press, New York, NY.

Hasegawa, I., F. Shinmachi, A. Noguchi y J. Yazaki. 2001. Physiological characterisation of root cell $\mathrm{Cd}^{2+} \mathrm{ab}-$ sorption and translocation to shoot in Brassica. pp. 448-449. En: Horst, W.J., M.K. Schenk, A. Bürkert, N. Claassen, H. Flessa, W.B. Frommer, H. Goldbach, H.W. Olfs, V. Römheld, B. Sattelmacher, U. Schmidhalter, S. Schubert, N. v. Wirén y L. Witenmayer (eds.). Plant nutrition-food security and sustainability of gro-ecosystems through basic and applied research. Kluwer, Dordrecht, The Netherlands.

Hijri, M. y I. Sanders . 2005. Low gene copy number shows that arbuscular mycorrhizal fungi inherit genetically different nuclei. Nature 433, 160-163.

Hildebrandt, U., M. Regvar y H. Bothe. 2007. Arbuscular mycorrhiza and heavy metal tolerance. Phytochem. 68, 139-146.

Husband, R., E. Herre y J. Young. 2002. Temporal variation in the arbuscular mycorrhizal communities colonizing seedlings in a tropical forest. FEMS Microbiol. Ecol. 42, 131-136.

Janoušková, M., D. Pavlíková y M. Vosátka. 2006. Potential contribution of arbuscular mycorrhiza to cadmium immobilisation in soil. Chemosphere 65, 1959-1965.

Janviera, C., F. Villeneuvea, C. Alabouvetteb, V. EdelHermannb, T. Mateille y C. Steinberg. 2007. Soil health through soil disease suppression: Which strategy from descriptors to indicators?. Soil Biol. Biochem. 39, 1-23.
Johnson, N., D. Tilman y D. Wedin. 1992. Plant and soil controls on mycorrhizal fungal communities. Ecol. 73, 2034-2042.

Ker, K. y C. Charest. 2010. Nickel remediation by AMcolonized sunflower. Mycorrhiza 20, 399-406.

Kernaghan, G. 2005. Mycorrhizal diversity: Cause and effect? Pedobiologia 49, 511-520.

Khan, A.G. 2001. Relationships between chromium biomagnification ratio, accumulation factor, and mycorrhizae in plants growing on tannery effluentpolluted soil. Environ. Int. 26, 417-423.

Khan, A.G. 2005. Role of soil microbes in the rhizosphere of plant growing on trace metal contaminated soils in phytoremediation. Trace Elements Medicine Biol. 355-364.

Kochian, L.V. 2001. Aluminium and heavy metal toxicity and resistance - Lessons to be learnt from similarities and differences. pp. 442-443. En: Horst, W.J., M.K. Schenk, A. Bürkert, N. Claassen, H. Flessa, W.B. Frommer, H. Goldbach, H.W. Olfs, V. Römheld, B. Sattelmacher, U. Schmidhalter, S. Schubert, N. v. Wirén y L. Witenmayer (eds.). Plant nutrition-food security and sustainability of groecosystems through basic and applied research. Kluwer, Dordrecht, The Netherlands.

Koske R.E. y J.N. Gemma. 1992. Fungal reactions to plants prior to mycorrhizal formation. pp. 3-36. En: Allen, M. F. (ed.). Mycorrhizal functioning: an integrated plant-fungal process. Chapman and Hall. New York, NY.

Kuhn. G., M. Hijri e I. Sanders. 2001. Evidence for the evolution of multiple genomes in arbuscular mycorrhizal fungi. Nature 414, 745-748.

Larcher, W. 2003. Physiological plant ecology. $4^{\text {th }}$ edition. Springer, Berlín.

Liao, J. P., X. G. Lin, Z. H. Cao, Y.Q. Shi y M.H. Wong. 2003. Interaction between arbuscular micorrhizae and heavy metals under sand culture experiments. Chemosphere 50, 847-853.

Lodge, D. J. 1989. The influence of soil moisture and flooding on formation of VA-endo and ectomycorrhizae in Populus and Salix. Plant Soil 117, 243-253.

Matsubara, Y. y H. Harada. 1996. Effect of constant and diurnally fluctuating temperatures on arbuscular mycorrhizal fungus infection and growth of infected asparagus (Asparagus officinalis L.) seedlings. J. Jpn. Soc. Hort. Sci. 65, 565-570.

Miller, S.P. 2000. Arbuscular mycorrhizal colonization of semi-aquatic grasses along a wide hydrologic gradient. New Phytol. 145, 145-155. 
Miller, S.P. y R.R. Sharitz. 2000. Manipulation of flooding and arbuscular mycorrhizal formation influences growth and nutrition of two semi-aquatic species. Funct. Ecol. 14, 738-748.

Miranda, D., C. Carranza y G. Fischer. 2008a. Calidad del agua de riego en la sabana de Bogotá, Unibiblos, Universidad Nacional de Colombia, Bogotá.

Miranda, D., C. Carranza, C.A. Rojas, C. M. Jerez, G. Fischer y J. Zurita. 2008b. Acumulación de metales pesados en suelos y plantas de cuatro cultivos hortícolas, regados con agua del río Bogotá. Rev. Colomb. Cienc. Hortíc. 2(2), 180-191.

Morton, J. 1988. Taxonomy of VA mycorrhizal fungi: Classification, nomenclature, and identification. Mycotaxon 32, 267-324.

Ortega-Larocea, M.P., C. Siebe, A. Estrada y R. Webster. 2007. Mycorrhiza inoculum potential of arbuscular mycorrhizal fungi in soils irrigated with waste water for various lengths of time, as affected by heavy metals and available P. Appl. Soil Ecol. 37, 129-138.

Pawlowska, T. y J.W. Taylor. 2004. Organization of genetic variation in individuals of arbuscular mycorrhizal fungi. Nature 427, 733-737.

Poschenrieder, C., M. Llugany, J. Bech y J. Barceló. 2001. Coopper uptake and transport patterns in pseudometallophytes from soils with a large range of $\mathrm{Cu}$ concentrations. pp. 458-459. En: Horst, W.J., M.K. Schenk, A. Bürkert, N. Claassen, H. Flessa, W.B. Frommer, H. Goldbach, H.W. Olfs, V. Römheld, B. Sattelmacher, U. Schmidhalter, S. Schubert, N. v. Wirén y L. Witenmayer (eds.). Plant nutritionfood security and sustainability of gro-ecosystems through basic and applied research. Kluwer, Dordrecht, The Netherlands.

Poschenrieder, C., J. Barceló. 2003. Capítulo 14: Estrés por metales pesados. pp 413-442. En: Reigosa, M.J., N. Pedrol y A. Sánchez Moreiras (eds.). La ecofisiología vegetal: Una ciencia de síntesis. Thompson, Madrid.

Powell, J.R., J.L. Parrent, M.M. Hart, J.N. Klironomos, M.C. Rilling y H. Maherali. 2009. Phylogenetic trait conservatism and the evolution of functional trade-offs in arbuscular mycorrhizal fungi. Proc. R. Soc. B 276, 4237-4245.

Read, D.J., H.K. Koucheki y J. Hodgson. 1976. Vesicular arbuscular mycorrhiza in natural systems. I. The occurrence of infection. New Phytol. 77, 641-653.

Redecker, D. 2008. Glomeromycota. Arbuscular mycorrhizal fungi and their relative(s). Version 14 January 2008. http://tolweb.org/Glomeromyco$\mathrm{ta} / 28715 / 2008.01 .14$. En: The Tree of Life Web Project, http://tolweb.org; consulta: octubre de 2010.
Remy, W., T.N. Taylor, H. Hass y H. Kerp. 1994. Four hundred million year old vesicular arbuscular mycorrhizae. Proc. Natl. Acad. Sci. USA 91, 11841-11843.

Rodríguez, D. 1991.Toxicocinética. pp. 11-14. En: Níquel. Centro Panamericano de Ecología Humana y Salud. México D.F.

Rodríquez-Serrano, M., N. Martínez de la Casa, M.Romero-Puertas, L.A. del Río, L.M. Sandalio.2008. Toxicidad del Cadmio en Plantas. Ecosistemas 17(3), 139-146.

Saldivar, L., A. Tovar y T. Fortoul. 1997a. Capítulo 13: Cadmio. pp. 211-226. En: Palacios y América. Introducción a la toxicología ambiental. ECO, Metepec, México, D.F.

Saldivar, L., A. Tovar y D. Guerrero. 1997b. Capítulo 11: Plomo. pp. 177-196. En: Palacios y América. Introducción a la toxicología ambiental. ECO, Metepec. México, D.F.

Sanders, I. y D. Croll. 2010. Genetics of the arbuscular mycorrhizal symbiosis. Ann. Rev. Gen. 44, 271-292.

Schenck, N.C., S.O. Graham y N.E. Green. 1975. Temperature and light effect on contamination and spore germination of vesicular-arbuscular mycorrhizal fungi. Mycologia 67, 1189-1192.

Schloter, M., O. Dilly y J.C. Munch. 2003. Indicators for evaluating soil quality. Agric. Ecosyst. Environ. 98, 255-262.

Schüßler A., D. Schwarzott y C. Walker. 2001. A new fungal phylum, the Glomeromycota: phylogeny and evolution. Mycological Res. 105, 1413-1421.

Serralde, A. M. y M. Ramírez. 2004. Análisis de poblaciones de micorrizas en maíz (Zea Mayz L.) cultivado en suelos ácidos bajo diferentes tratamientos agronómicos. Revista Corpoica 5 (1), 31-40.

Sharma, Py R.Dubey. 2005. Lead toxicity in plants. Braz J. Plant Physiol. 17(1), 35-52.

Sharma, S., D. Pant, S. Singh, R. Sinha y A. Adholeya. 2007. Chapter 7: Mycorrizae in Indian Agriculture. pp. 239-291. Effect of pollution and metal toxicity on Mycorrizal colonization and plant growth. En: Hamel, C., C. Plenchette (eds.). Mycorrhizae in crop production. Haworthpress, New York, NY.

Smith, S., E. Facelli, S. Pope y F. Smith. 2010. Plant performance in stressful environments: interpreting new and established knowledge of the roles of arbuscular mycorrhizas. Plant Soil 326, 3-20.

Sylvia, D., J. Fuhrmannn, P. Hartel y D. Zuberer. 2005. Principles and applications of soil microbiology. Pearson-Prentice Hall, New Jersey, NJ. 
Takács, T., B. Biró e I. Vörös. 2001. Arbuscular mycorrhizal effect on heavy metal uptake of ryegrass (Lolium perenne L.) in pot culture with polluted soils. pp. 480-481. En: Horst, W.J., M.K. Schenk, A. Bürkert, N. Claassen, H. Flessa, W.B. Frommer, H. Goldbach, H.W. Olfs, V. Römheld, B. Sattelmacher, U. Schmidhalter, S. Schubert, N. v. Wirén y L. Witenmayer (eds.). Plant nutrition-food security and sustainability of gro-ecosystems through basic and applied research. Kluwer, Dordrecht, The Netherlands.

Unz, R.F. y K.L. Shuttleworth. 1996. Microbial mobilization and immobilization of heavy metals. Environ. Biotechnol. 7, 307-310.

Vandenkoornhuyse, P., R. Husband, T. Daniell, I. Watson, J. Duck y J. Fitter. 2002. Arbuscular mycorrhizal community composition associated with two plant species in a grassland ecosystem. Mol. Ecol.11, 1555-1564.

Verkleij, J.A.C., N.A.L.M van Hoof, A.N. Chardonnens, P.L.M. Koevoets, H. Hakvoort, W.M ten Bookum, H. Schat y W.H.O. Ernst. 2001. Mechanism of heavy metal resistance in Silene vulgaris. pp. $446-$ 447. En: Horst, W.J., M.K. Schenk, A. Bürkert, N. Claassen, H. Flessa, W.B. Frommer, H. Goldbach, H.W. Olfs, V. Römheld, B. Sattelmacher, U. Schmidhalter, S. Schubert, N. v. Wirén y L. Witenmayer (eds.). Plant nutrition-food security and sustainability of gro-ecosystems through basic and applied research. Kluwer, Dordrecht, The Netherlands.

Vega, S. 1988. Capítulo 6: Metales. pp. 127-180. En: Evaluación epidemiológica de riesgos causados por agentes químicos ambientales. Ministerio de Salud y Acción Social. Dirección Nacional de Calidad Ambiental, Buenos Aires.

Vierheilig, H. y Y. Piché. 2002. Signaling in arbuscular mycorrhiza: facts and hypotheses. En: Manthey J. y B. Buslig (eds.). Flavonoids in the living system. Plenum Press. New York, NY.

Vogel-Mikǔs, K., P. Pongrac, P. Kump, M. Nečemer y M. Regvar. 2006. Colonisation of a $\mathrm{Zn}, \mathrm{Cd}$ and $\mathrm{Pb}$ hy- peraccumulator Thalpsi praecox Wulfen with indigenous-arbuscular mycorrhizal fungi mixture induce changes in heavy metal and nutrient uptake. Environ. Pollution 139, 362-371.

Vörös, I. y T. Takács. 2001. The effect of different strains of AMF on growth and heavy metal uptake of cucumber (Cucumis sativus). pp. 478-479. En: Horst, W.J., M.K. Schenk, A. Bürkert, N. Claassen, H. Flessa, W.B. Frommer, H. Goldbach, H.W. Olfs, V. Römheld, B. Sattelmacher, U. Schmidhalter, S. Schubert, N. v. Wirén y L. Witenmayer (eds.). Plant nutrition-food security and sustainability of groecosystems through basic and applied research Kluwer, Dordrecht, The Netherlands.

Walker, C. 1983. Taxonomic concepts in the Endogonaceae: spore wall characteristics in species descriptions. Mycotaxon 18, 443-455.

Wang, J. y C. Chen. 2009. Biosorbents for heavy metals removal and their future. Biotechnol. Adv. 27, 195-226.

Xin, J., B. Huang, Z. Yang, J. Yuan, H. Dai y Q. Qiu. 2010. Responses of different water spinach cultivars and their hybrid to $\mathrm{Cd}, \mathrm{Pb}$ and $\mathrm{Cd}-\mathrm{Pb}$ exposures. J. Hazardous Materials 175, 468-476.

Yadav, S.K. 2010. Heavy metals toxicity in plants: An overview on the role of glutathione and phytochelatins in heavy metal stress tolerance of plants. South African Bot. 76, 167-179.

Yang, X. E., M. J. Yang, Z. Y. Shen, J. R. Bacon, E. Paterson y J. M. Wilson. 2001. Some mechanisms of zinc and cadmium detoxification in a zinc and cadmium hyper-accumulation plant species (Thlaspi) pp. 444-445. En: Horst, W.J., M.K. Schenk, A. Bürkert, N. Claassen, H. Flessa, W.B. Frommer, H. Goldbach, H.W. Olfs, V. Römheld, B. Sattelmacher, U. Schmidhalter, S. Schubert, N. v. Wirén y L. Witenmayer (eds.). Plant nutrition-food security and sustainability of gro-ecosystems through basic and applied research. Kluwer, Dordrecht, The Netherlands. 